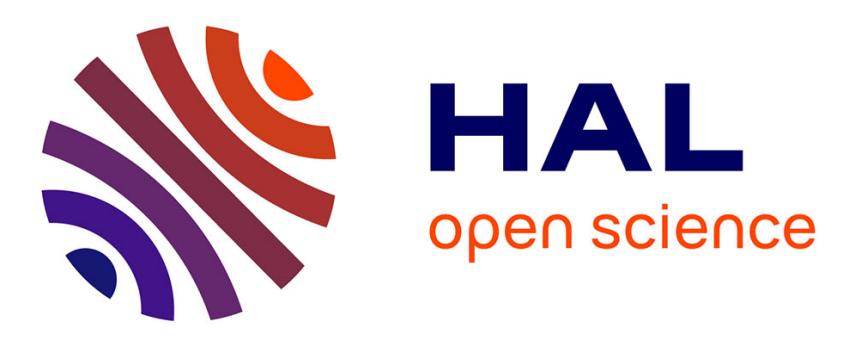

\title{
Data and model uncertainties in complex aerospace engineering systems
}

Evangéline Capiez-Lernout, M. Pellissitti, H. Pradlwarter, G.I. Schueller, Christian Soize

\section{- To cite this version:}

Evangéline Capiez-Lernout, M. Pellissitti, H. Pradlwarter, G.I. Schueller, Christian Soize. Data and model uncertainties in complex aerospace engineering systems. Journal of Sound and Vibration, 2006, 295 (3-5), pp.923-938. 10.1016/j.jsv.2006.01.056 . hal-00686152

\section{HAL Id: hal-00686152 \\ https://hal.science/hal-00686152}

Submitted on 7 Apr 2012

HAL is a multi-disciplinary open access archive for the deposit and dissemination of scientific research documents, whether they are published or not. The documents may come from teaching and research institutions in France or abroad, or from public or private research centers.
L'archive ouverte pluridisciplinaire HAL, est destinée au dépôt et à la diffusion de documents scientifiques de niveau recherche, publiés ou non, émanant des établissements d'enseignement et de recherche français ou étrangers, des laboratoires publics ou privés. 


\section{Data and model uncertainties in complex aerospace engineering systems.}

Capiez-Lernout, E. ${ }^{1, a, \star}$, Pellissetti, M. ${ }^{2, b}$, Pradlwarter, H. ${ }^{3, b}$, Schueller, G.I. ${ }^{4, b}$, Soize,C. ${ }^{5, a}$

${ }^{1}$ Evangeline.Capiez-Lernout@univ-mlv.fr

${ }^{2}$ Manuel.Pellissetti@uibk.ac.at

${ }^{3}$ Helmut.Pradlwarter@uibk.ac.at

${ }^{4}$ G.I.Schueller@uibk.ac.at

${ }^{5}$ Christian.Soize@univ-mlv.fr

${ }^{a}$ Laboratoire de Mécanique

University of Marne-La-Vallée

5, Boulevard Descartes,

F-77455 Marne-La-Vallée

Tel: 0033160957798

Fax:0033160957799.

${ }^{b}$ Institute of Engineering Mechanics

Leopold-Franzens University

A-6020, Innsbruck.

${ }^{\star}$ Corresponding author. 


\begin{abstract}
Parametric probabilistic approaches allow data uncertainties to be modeled, but have some difficulties to represent model uncertainties. It has been recently shown that both model and data uncertainties can be taken into account with a non-parametric approach. Moreover, it is known that with increasing complexity of a mechanical system, model uncertainties also increase. Based on these considerations, both parametric and non-parametric probabilistic approaches are used on a complex system of aerospace engineering constituted of a satellite coupled with its launcher. First, a parametric probabilistic model is constructed for analysing the sensitivity of the response due to data uncertainties. Then, the non-parametric probabilistic model is introduced with the same uncertainty level in order to study the sensitivity of the response with respect to the model and the data uncertainties. The dynamical responses obtained with these two probabilistic approaches are analysed in order to quantify the sensitivity of the structure to data uncertainties as well as model uncertainties.
\end{abstract}

Key words:

Structural dynamics, complex structures, random uncertainties, parametric probabilistic model, non-parametric probabilistic model.

\title{
1 Introduction
}

In structural dynamics, numerical models are used to perform dynamic analyses of complex structural components of mechanical systems. Every manufacturing process induces physical discrepancies. Consequently, the manufactured system is different from the designed system. These differences can have significant effects on the dynamics of the structure. In order to construct predictive numerical models, a mechanical-numerical model is constructed from the designed system using the finite element method. Such a mechanical-numerical model will be called here the mean finite element model of the structure. It should be noted that such a deterministic model is usually not sufficient for a robust prediction of the dynamic response of the structure. In order to increase the robustness of the predictions, this mean model is used for implementing probabilistic models for taking into account uncertainties. In particular, the 
robustness of the predictions are of great relevance to many industrial areas such as aerospace industry.

The uncertainties can then be splitted in two complementary classes. The first one refers the uncertain parameters in the mean finite element model describing data uncertainties. The second class is due to simplifications introduced in the construction of the mean finite element model using the mathematical-mechanical-numerical modelling process, which cannot be taken into account by the uncertain parameters of the mean finite element model. In this paper, two probabilistic approaches for modelling random uncertainties are considered, namely the parametric probabilistic approach for data uncertainties in the mean finite element model and the non-parametric probabilistic approach for model uncertainties with respect to the mean finite element model.

The parametric probabilistic approach allows data uncertainties to be modelled by considering the uncertain parameters of the mean finite element model as random quantities. Such uncertain parameters are usually the geometrical parameters, the parameters of the constitutive equations and the boundary conditions. As a consequence, each random finite element matrix results from a deterministic mapping of the random parameters. It should be noted that parametric approaches are recognised as efficient methods for modelling data uncertainties and are widely used in computational mechanics (see for instance [1-7]).

Nevertheless, the parametric probabilistic approach has difficulties to take into account model uncertainties. For instance, when dealing with a beam structure, the use of the Euler beam theory instead of the tridimensional elasticity theory corresponds to the introduction of a reduced admissible displacement field (reduced kinematics). An approach, called the non-parametric probabilistic approach of model uncertainties, has recently been introduced to take into account model uncertainties. The theoretical concepts have been introduced and developed in [8,9] and several experimental validations [10-13] and numerical validations $[14,15]$ have been carried out. In this paper, the vibration amplitudes are assumed to be sufficiently small in order that linearised equations can be used. For the vibration problem considered, the existence of local non-linearities at certain junctions of the structure can be assumed to be negligible in the frequency band of analysis. The non-parametric probabilistic approach of model uncertainties 
proposed here is then implemented in a linear context. Such a probabilistic approach allows the robustness of a linear numerical model with respect to model uncertainties for the set of all the possible linear models to be analysed. With such a non-parametric probabilistic approach, the generalised matrices issued from a mean reduced matrix model of the structure are replaced by random matrices. The probability distributions of these random matrices are constructed by using the maximum entropy principle under constraints defined by the available information and yields a new class of random matrices $[8,9,14]$. With such a formulation, the global dispersion level of each random matrix is controlled by a unique positive parameter which is called the dispersion parameter. Moreover, it is important to note that this methodology can easily be extended to the case of nonhomogeneous random uncertainties [10] and to the case of mistuned cyclic structures with random uncertainties $[16,17]$ for which dynamical substructuring methods are required and used. Furthermore, it has been shown (see for instance $[14,13,15])$ that the non-parametric probabilistic approach can represent model uncertainties. In view of these results, it would be very interesting to apply the parametric probabilistic approach and the nonparametric probabilistic approach for a complex structure for which the mean finite element model is simplified. Since the parametric probabilistic approach allows data uncertainties to be modeled and since the non-parametric probabilistic approach takes into account the model uncertainties, the main objective of this paper is to show the role played by data uncertainties and by model uncertainties in a complex dynamical system.

In section 2 , the dynamical equations issued from the parametric probabilistic approach are presented. Section 3 is devoted to the dynamical equations issued from the non-parametric probabilistic approach. Section 4 deals with the identification of the dispersion parameters of the non-parametric probabilistic approach with respect to the parametric probabilistic approach in order to introduce a similar level of uncertainty for each probabilistic approach. The methodology used for solving the random equations is then presented in section 5. Finally, Section 6 is devoted to the analysis of a complex aerospace engieering system. The system considered is a satellite of the European Space Agency, whose mean model is a large tridimensional finite element model. Both parametric and non-parametric probabilistic approaches are used for modelling data uncertainties and model uncertainties in the satellite. Dynamical analyses are carried out with a similar level of uncertainty for each probabilistic model. Convergence analyses are 
systematically performed with respect to the dimension of the reduced model and the number of realizations for statistical estimations. Two cases are considered: the free satellite and the satellite coupled with the launcher which is considered as deterministic in the present work.

\subsection{Mathematical notation conventions}

In this paper, the following conventions are adopted:

(1) A real or complex deterministic scalar is denoted by a lower case letter (for instance $f$ )

(2) A real or complex-valued random variable is denoted by an upper case letter (for instance F)

(3) A real or complex deterministic vector is denoted by a boldface lower case letter (for instance $\left.\mathbf{f}=\left(f_{1}, \ldots, f_{n}\right)\right)$

(4) A real or complex-valued random vector is denoted by a boldface upper case letter (for instance $\left.\mathbf{F}=\left(F_{1}, \ldots, F_{n}\right)\right)$

(5) A real or complex deterministic matrix is denoted by an upper case letter between brackets (for instance $[A]$ )

(6) A real or complex-valued random matrix is denoted by a boldface upper case letter between brackets (for instance $[\mathbf{A}]$ )

(7) All the deterministic quantities related to the mean model are underlined (for instance $\underline{f}, \underline{\mathbf{f}},[\underline{A}])$ 


\section{Random response of the dynamical system with data uncertainties modeled by the parametric probabilistic approach}

\subsection{Mean finite element model of the dynamical system}

We are interested in the linear vibrations of a lightly damped free structure around a static equilibrium configuration considered as a natural state without prestresses in the low frequency band $\mathbb{B}$. For all $\omega$ in band $\mathbb{B}$, the mean finite element matrix equation of the structure is written as

$$
\left(-\omega^{2}[\underline{M}]+i \omega[\underline{D}]+[\underline{K}]\right) \underline{\mathbf{u}}(\omega)=\underline{\mathbf{f}}(\omega),
$$

in which $\underline{\mathbf{u}}(\omega)$ and $\underline{\mathbf{f}}(\omega)$ are the $\mathbb{C}^{m}$-vectors of the DOFs and of the external forces. Since the structure has a free boundary, the mean mass matrix $[\underline{M}]$ is a positive-definite symmetric $(m \times$ $m$ ) real matrix and the mean damping and stiffness matrices are positive semidefinite symmetric $(m \times m)$ real matrices. Furthermore, it is assumed that the kernel of mean matrices $[\underline{D}]$ and $[\underline{K}]$ is identical, constituted of $r$ rigid-body modes with $0 \leq r \leq 6$ and denoted as $\underline{\varphi}_{1}, \ldots, \underline{\varphi}_{r}$.

\subsection{Parametric model of random uncertainties}

Let $\underline{\mathbf{x}}=\left(\underline{x}_{1}, \ldots, \underline{x}_{\mu}\right)$ be the $\mathbb{R}^{\mu}$-vector whose components describe mechanical parameters such as geometric parameters of the structure, coefficients of the elasticity tensor, mass density,etc. Consequently, the finite element mass, damping and stiffness matrices are considered as a function of these parameters. Since these parameters are uncertain, one then introduces the $\mathbb{R}^{\mu}$-valued random variable $\mathbf{X}=\left(X_{1}, \ldots, X_{\mu}\right)$. The random finite element model is then written as

$$
\left(-\omega^{2}\left[\mathbf{M}^{\mathrm{par}}\right]+i \omega\left[\mathbf{D}^{\mathrm{par}}\right]+\left[\mathbf{K}^{\mathrm{par}}\right]\right) \mathbf{U}^{\mathrm{par}}(\omega)=\underline{\mathbf{f}}(\omega)
$$

in which $\mathbf{U}^{\mathrm{par}}(\omega)$ is the $\mathbb{C}^{m}$-valued random vector of the DOFs and where $\left[\mathbf{M}^{\mathrm{par}}\right]=[M(\mathbf{X})]$, and $\left[\mathbf{D}^{\mathrm{par}}\right]=[D(\mathbf{X})],\left[\mathbf{K}^{\mathrm{par}}\right]=[K(\mathbf{X})]$ are the random finite element mass and damping, stiffness matrices with values in the set of the positive-definite symmetric $(m \times m)$ real matrices and in the set of the positive semidefinite symmetric $(m \times m)$ real matrices. The components 
$\left\{X_{i}, i \in\{1, \ldots, \mu\}\right\}$ of random vector $\mathbf{X}$ are independent Gaussian random variables of mean value $m_{i}=\underline{x}_{i}$ and of standard deviation $\sigma_{i}$. A numerical solver adapted to the Monte Carlo numerical simulation and using a reduced model constructed with random modal analysis [18$21]$ is used for calculating the random elastic response.

\section{Random response of the dynamical system with model and data uncertainties modeled by the non-parametric probabilistic approach}

Let us recall that the main idea of the non-parametric probabilistic approach of model and data uncertainties consists in replacing the generalised matrices of a mean reduced matrix model of the structure by random matrices whose probability model is constructed with the maximum entropy principle. In particular, the theoretical construction and the physical concepts of this recent probabilistic approach are detailed in $[8,9,14,13]$. In this Section, the main steps for establishing the random equations are summarised.

\subsection{Mean reduced matrix model}

Since one is interested in the elastic motion of the structure, one then introduces the $(m \times n)$ real matrix $[\underline{\Phi}]$ whose columns are the $n \lll m$ eigenvectors $\underline{\varphi}_{\alpha}$ related to the $n$ strictly positive lowest eigenfrequencies $\underline{\lambda}_{\alpha}=\underline{\omega}_{\alpha}^{2}$. The mean reduced matrix model is written as

$$
\underline{\mathbf{u}}(\omega)=[\underline{\Phi}] \underline{\mathbf{q}}(\omega),
$$

in which $\underline{\mathbf{q}}(\omega)$ is the $\mathbb{C}^{n}$-vector of the generalised coordinates solution of the mean reduced equation

$$
\left(-\omega^{2}\left[\underline{M}_{\mathrm{red}}\right]+i \omega\left[\underline{D}_{\mathrm{red}}\right]+\left[\underline{K}_{\mathrm{red}}\right]\right) \underline{\mathbf{q}}(\omega)=\underline{\mathcal{F}}(\omega),
$$

in which $\underline{\mathcal{F}}(\omega)=[\underline{\Phi}]^{T} \underline{\mathbf{f}}(\omega)$ is the $\mathbb{C}^{n}$-vector of the generalised forces and where the mean reduced mass, damping and stiffness matrices $\left[\underline{M}_{\mathrm{red}}\right]=[\underline{\Phi}]^{T}[\underline{M}][\underline{\Phi}],\left[\underline{D}_{\mathrm{red}}\right]=[\underline{\Phi}]^{T}[\underline{D}][\underline{\Phi}]$ and $\left[\underline{K}_{\mathrm{red}}\right]=[\underline{\Phi}]^{T}[\underline{K}][\underline{\Phi}]$ are positive-definite symmetric $(n \times n)$ real matrices. 
The use of the non-parametric probabilistic approach of model and data uncertainties yields the random matrix equation

$$
\left(-\omega^{2}\left[\mathbf{M}_{\mathrm{red}}^{\mathrm{npar}}\right]+i \omega\left[\mathbf{D}_{\mathrm{red}}^{\mathrm{npar}}\right]+\left[\mathbf{K}_{\mathrm{red}}^{\mathrm{npar}}\right]\right) \mathbf{Q}(\omega)=\underline{\mathcal{F}}(\omega)
$$

in which $\left[\mathbf{M}_{\mathrm{red}}^{\mathrm{npar}}\right]$, $\left[\mathbf{D}_{\mathrm{red}}^{\mathrm{npar}}\right]$ and $\left[\mathbf{K}_{\mathrm{red}}^{\mathrm{npar}}\right]$ are positive-definite symmetric $(n \times n)$ real-valued matrices corresponding to the random reduced mass, damping and stiffness matrices and where $\mathbf{Q}(\omega)$ is the $\mathbb{C}^{n}$-valued random vector of the random generalised coordinates. The $\mathbb{C}^{m}$-valued random vector $\mathbf{U}^{\text {npar }}(\omega)$ is thus reconstructed by

$$
\mathbf{U}^{\mathrm{npar}}(\omega)=\left[\underline{\Phi}^{n}\right] \mathbf{Q}(\omega)
$$

\subsubsection{Probability distributions of the random matrices}

The positive-definite random matrices $\left[\mathbf{M}_{\mathrm{red}}^{\mathrm{npar}}\right],\left[\mathbf{D}_{\mathrm{red}}^{\mathrm{npar}}\right]$ and $\left[\mathbf{K}_{\mathrm{red}}^{\mathrm{npar}}\right]$ are written as

$$
\begin{aligned}
{\left[\mathbf{M}_{\mathrm{red}}^{\mathrm{npar}}\right] } & =\left[\underline{L}_{M}\right]^{T}\left[\mathbf{G}_{M}\right]\left[\underline{L}_{M}\right] \\
{\left[\mathbf{D}_{\mathrm{red}}^{\mathrm{npar}}\right] } & =\left[\underline{L}_{D}\right]^{T}\left[\mathbf{G}_{D}\right]\left[\underline{L}_{D}\right] \\
{\left[\mathbf{K}_{\mathrm{red}}^{\mathrm{npar}}\right] } & =\left[\underline{L}_{K}\right]^{T}\left[\mathbf{G}_{K}\right]\left[\underline{L}_{K}\right],
\end{aligned}
$$

in which $\left[\underline{L}_{M}\right],\left[\underline{L}_{D}\right]$ and $\left[\underline{L}_{K}\right]$ are diagonal $(n \times n)$ real matrices such that $\left[\underline{M}_{\mathrm{red}}\right]=\left[\underline{L}_{M}\right]^{T}\left[\underline{L}_{M}\right]$, $\left[\underline{D}_{\mathrm{red}}\right]=\left[\underline{L}_{D}\right]^{T}\left[\underline{L}_{D}\right]$ and $\left[\underline{K}_{\mathrm{red}}\right]=\left[\underline{L}_{K}\right]^{T}\left[\underline{L}_{K}\right]$. The probability distribution of random matrices $\left[\mathbf{G}_{M}\right],\left[\mathbf{G}_{D}\right]$ and $\left[\mathbf{G}_{K}\right]$ is derived from the maximum entropy principle issued from the information theory [22] with the available information [8]. It is shown in [8,9] that random matrices $\left[\mathbf{G}_{M}\right],\left[\mathbf{G}_{D}\right]$ and $\left[\mathbf{G}_{K}\right]$ are independent random variables whose dispersion level can be controlled by the positive real parameters $\delta_{M}, \delta_{D}$ and $\delta_{K}$ which are independent of the dimension $n$.

Below, $[\mathbf{G}]$ denotes $\left[\mathbf{G}_{M}\right],\left[\mathbf{G}_{D}\right]$ or $\left[\mathbf{G}_{K}\right]$ and $\delta$ denotes the corresponding dispersion parameter. The probability density function of random matrix $[\mathbf{G}]$ with respect to the volume element

$$
\tilde{d} G=2^{n(n-1) / 4} \prod_{1 \leq i \leq j \leq n} d G_{i j}
$$


is written as

$$
p_{[\mathbf{G}]}([G])=\mathbb{1}_{\mathbb{M}_{n}^{+}(\mathbb{R})}([G]) \times C_{G} \times(\operatorname{det}([G]))^{\left(1-\delta^{2}\right)\left(2 \delta^{2}\right)^{-1}(n+1)} \times e^{-(n+1)\left(2 \delta^{2}\right)^{-1} \operatorname{tr}[G]},
$$

in which $\mathbb{1}_{\mathbb{M}_{n}^{+}(\mathbb{R})}$ denotes the indicatrix function of the set of all the symmetric $(n \times n)$ real positive-definite matrices, det is the determinant, $\operatorname{tr}$ is the trace and where $C_{G}$ is the positive constant such that

$$
C_{G}=\frac{(2 \pi)^{-n(n-1) / 4}\left(\frac{n+1}{2 \delta^{2}}\right)^{n(n+1)\left(2 \delta^{2}\right)^{-1}}}{\prod_{j=1}^{n} \Gamma\left(\frac{n+1}{2 \delta^{2}}+\frac{1-j}{2}\right)}
$$

in which $\Gamma(z)$ is the gamma function defined for all $z>0$ by $\Gamma(z)=\int_{0}^{\infty} t^{z-1} e^{-t} d t$. Eq. (11) shows that the entries $[\mathbf{G}]_{j k}$ of the random matrix $[\mathbf{G}]$ are dependent random variables.

The following algebraic representation of the random positive-definite symmetric real matrix $[\mathbf{G}]$ allows a procedure for the Monte Carlo numerical simulation of the random matrix $[\mathbf{G}]$ to be defined. The random matrix $[\mathbf{G}]$ is written as

$$
[\mathbf{G}]=\left[\mathbf{L}_{G}\right]^{T}\left[\mathbf{L}_{G}\right],
$$

in which $\left[\mathbf{L}_{G}\right]$ is an $(n \times n)$ upper triangular random matrix resulting from the Cholesky factorisation such that

(1) the random variables $\left\{\left[\mathbf{L}_{G}\right]_{j j^{\prime}}, j \leq j^{\prime}\right\}$ are independent;

(2) for $j<j^{\prime}$, the real-valued random variable $\left[\mathbf{L}_{G}\right]_{j j^{\prime}}$ can be written as $\left[\mathbf{L}_{G}\right]_{j j^{\prime}}=\sigma_{n} U_{j j^{\prime}}$ in which $\sigma_{n}=\delta(n+1)^{-1 / 2}$ and where $U_{j j^{\prime}}$ is a real-valued Gaussian random variable with zero mean and variance equal to 1 ;

(3) for $j=j^{\prime}$, the positive-valued random variable $\left[\mathbf{L}_{G}\right]_{j j}$ can be written as $\left[\mathbf{L}_{G}\right]_{j j}=\sigma_{n} \sqrt{2 V_{j}}$ in which $\sigma_{n}$ is defined above and where $V_{j}$ is a positive-valued gamma random variable whose probability density function $p_{V_{j}}(v)$ with respect to $d v$ is written as

$$
p_{V_{j}}(v)=\mathbb{1}_{\mathbb{R}^{+}}(v) \frac{1}{\Gamma\left(\alpha_{n, j}\right)} v^{\alpha_{n, j}-1} e^{-v} \quad, \quad \alpha_{n, j}=\frac{n+1}{2 \delta^{2}}+\frac{1-j}{2}
$$




\section{Identification of the dispersion parameters of the non-parametric approach with re- spect to the parametric approach}

Let us recall that the main objective of thie paper is to analyse the role played by data uncertainties and by model uncertainties in the dynamical response of a complex mechanical system. The parametric probabilistic approach allows data uncertainties to be analysed while the nonparametric probabilistic approach is proposed to analyse model and data uncertainties. In order to quantify the role played by each one of the two kinds of uncertainties, it is necessary to introduce the same level of uncertainties in the two probabilistic approaches. It is assumed that the probability distributions of uncertain data (parametric probabilistic approach) are given. Then the level of uncertainties of the non-parametric probabilistic approach has to be defined with respect to the level of uncertainties of the parametric probabilistic approach. It means that the dispersion parameters $\delta_{M}, \delta_{D}$ and $\delta_{K}$ which control the dispersion of each random matrix issued from the non-parametric probabilistic approach are then identified with respect to the parametric probabilistic approach. Since the robustness of any finite element model can be characterised by its lowest eigenfrequency, the method which is chosen to calibrate the mass and the stiffness dispersion parameters $\delta_{M}$ and $\delta_{K}$ of the non-parametric probabilistic approach, is the following.

Let $\Omega_{1}^{\text {par }}$ and $\Omega_{1}^{\text {npar }}$ be the non zero lowest eigenfrequencies of the dynamical system modeled with the parametric and the non-parametric probabilistic approaches respectively. The probability density functions of the random eigenfrequencies $\Omega_{1}^{\text {par }}$ and $\Omega_{1}^{\text {npar }}$ denoted by $p_{\Omega_{1}^{\text {par }}}$ and $p_{\Omega_{1}^{\text {nar }}}$ are then compared in the least square sense. The two-dimensional cost function $J\left(\delta_{M}, \delta_{K}\right)$ is then introduced such that

$$
J\left(\delta_{M}, \delta_{K}\right)=\frac{\left\|p_{\Omega_{1}^{\mathrm{npar}}}\left(\delta_{M}, \delta_{K}\right)-p_{\Omega_{1}^{\mathrm{par}}}\right\|_{\mathcal{L}^{2}}}{\| p_{\Omega_{1}^{\mathrm{par}}}||_{\mathcal{L}^{2}}},
$$

in which the norm $\|f\|_{\mathcal{L}^{2}}$ is given by

$$
\|f\|_{\mathcal{L}^{2}}=\left(\int_{\mathbb{R}}|f(\mathbf{x})|^{2} d \mathbf{x}\right)^{1 / 2}
$$

The identification is then carried out such that parameters $\delta_{M}$ and $\delta_{K}$ minimize the cost function, 
i.e., are solution of the optimization problem

$$
\min _{\delta_{M}, \delta_{K}} J\left(\delta_{M}, \delta_{K}\right)
$$

The dispersion parameter $\delta_{D}$ is identified by using the identification method proposed in [16]. Let $\left[\mathbf{D}_{\mathrm{red}}^{\mathrm{par}}\right]$ be the random generalized damping matrix of the parametric probabilistic approach. The dispersion parameter $\delta_{D}$ is then calculated by

$$
\delta_{D}=\sqrt{\frac{W_{D}^{\mathrm{par}}(n+1)}{\operatorname{tr}\left(\left[\underline{D}_{\mathrm{red}}\right]\right)^{2}+\operatorname{tr}\left(\left[\underline{D}_{\mathrm{red}}\right]^{2}\right)}},
$$

in which $W_{D}^{\mathrm{par}}$ is given by

$$
W_{D}^{\mathrm{par}}=E\left\{\left\|\left[\mathbf{D}_{\mathrm{red}}^{\mathrm{par}}\right]-\left[\underline{D}_{\mathrm{red}}\right]\right\|_{F}^{2}\right\}
$$

and where $\|[A]\|_{F}^{2}=\operatorname{tr}\left([A][A]^{T}\right)$.

\section{Methodology for solving the random equations and for analysing the random re- sponses}

\subsection{Convergence analysis of the stochastic system}

The Monte Carlo numerical simulation is carried out with $n_{s}$ realisations denoted by $\theta_{1}, \ldots, \theta_{n_{s}}$. The forced response of the stochastic dynamical system is studied in the low-frequency band B. The numerical values of the dispersion parameters $\delta_{M}, \delta_{D}$ and $\delta_{K}$ are obtained by using the identification procedure described in Section 4. For each realisation $\theta_{i}$, a sample of the random variable $U^{\text {npar }}\left(\theta_{i} ; \omega\right)$ is calculated in solving Eqs. (5) and (6). A stochastic convergence analysis is carried out in order to define the number $n$ of modes to be kept and the number $n_{s}$ of realisations used in the Monte Carlo numerical simulation. The second-order stochastic convergence is based on the use of the sequence ||$\left|\mathbf{U}^{\text {npar }}\right|||$ defined by

$$
\left\|\left|\mathbf{U}^{\mathrm{npar}}\right|\right\|^{2}=E\left\{\int_{\omega \in \mathbb{B}}\left\|\mathbf{U}^{\mathrm{npar}}(\omega)\right\|^{2} d \omega\right\}
$$


in which $\left\|\mathbf{U}^{\text {npar }}(\omega)\right\|^{2}$ is the hermitian norm of random vector $\mathbf{U}^{\text {npar }}(\omega)$. The convergence is then analysed in studying the function $\left(n_{s}, n\right) \mapsto \operatorname{Conv}\left(n_{s}, n\right)$ defined by

$$
\operatorname{Conv}\left(n_{s}, n\right)^{2}=\frac{1}{n_{s}} \sum_{j=1}^{n_{s}} \int_{\mathbb{B}}\left\|U^{\mathrm{npar}}\left(\omega, \theta_{j}\right)\right\|^{2} d \omega .
$$

\subsection{Confidence region of the random response}

The confidence region of the random response is constructed for a given probability level $P_{c}$. The following reasoning is valuable for both probabilistic approaches. The exponents ${ }^{\mathrm{npar}}$ or ${ }^{\mathrm{par}}$ are then omitted in the notations used below.

Let $j_{\text {obs }}$ be an observation node. Let $\underline{\mathbf{u}}_{j_{\text {obs }}}(\omega)$ be the $\mathbb{C}^{3}$-vector and let $\mathbf{U}_{j_{\text {obs }}}(\omega)$ be the $\mathbb{C}^{3}$ valued random variable related to the three translational DOFs of node $j_{\text {obs }}$. One then introduces the scalar $\underline{w}_{j_{\mathrm{obs}}}(\omega)=20 \log _{10}\left(\left\|\underline{\mathbf{u}}_{j_{\mathrm{obs}}}(\omega)\right\|\right)$ and the random variable $W_{j_{\mathrm{obs}}}(\omega)=$ $20 \log _{10}\left(\left\|\mathbf{U}_{j_{\mathrm{obs}}}(\omega)\right\|\right)$.

The mean value $W_{j_{\text {obs }}}^{0}(\omega)$ of the random response is then defined by

$$
W_{j_{\mathrm{obs}}}^{0}(\omega)=20 \log _{10}\left(\left|\mathcal{E}\left\{|| \mathbf{U}_{j_{\mathrm{obs}}}(\omega)||\right\}\right|\right)
$$

Let $\omega$ fixed in $\mathbb{B}$. The quantile function $Q_{W_{j_{\mathrm{obs}}}}(\alpha ; \omega)$ of the random variable $W_{j_{\mathrm{obs}}}(\omega)$ is defined by

$$
Q_{W_{j_{\mathrm{obs}}}}(\alpha ; \omega)=\inf _{w} F_{W_{j_{\mathrm{obs}}}}(w ; \omega) \geq \alpha,
$$

in which $F_{W_{j_{\mathrm{obs}}}}(w ; \omega)$ is the cumulative distribution function of the random variable $W_{j_{\mathrm{obs}}}(\omega)$. Let $\widetilde{W}_{j_{\mathrm{obs}}}\left(\theta_{1} ; \omega\right)<\ldots<\widetilde{W}_{j_{\mathrm{obs}}}\left(\theta_{n_{s}} ; \omega\right)$ be the order statistic associated with $W_{j_{\mathrm{obs}}}\left(\theta_{1} ; \omega\right), \ldots$, $W_{j_{\mathrm{obs}}}\left(\theta_{n_{s}} ; \omega\right)$. The unbiased estimation of cumulative distribution function $F_{W_{j_{\mathrm{obs}}}}(w ; \omega)$ is defined by

$$
\hat{F}_{W_{j_{\mathrm{obs}}, n_{s}}}(w ; \omega)=\frac{1}{n_{s}} \sum_{k=1}^{n_{s}} H^{0}\left(w-\widetilde{W}_{j_{\mathrm{obs}}}\left(\theta_{k} ; \omega\right)\right),
$$

in which $H^{0}$ is such that $H^{0}(x)=1$ if $x \geq 0$ and $H^{0}(x)=0$ if not. The upper envelope $w_{j_{\mathrm{obs}},+}$ and the lower envelope $w_{j_{\mathrm{ob}},-}$ delimiting the confidence region with probability level $P_{c}$ is then given by 


$$
\begin{aligned}
& w_{j_{\mathrm{obs}},+}(\omega)=\widetilde{W}_{j_{\mathrm{obs}}}\left(\theta_{k_{+}} ; \omega\right), k_{+}=\operatorname{fix}\left(0.5 n_{s}\left(1+P_{c}\right)\right) \\
& w_{j_{\mathrm{obs}},-}(\omega)=\widetilde{W}_{j_{\mathrm{obs}}}\left(\theta_{k_{-}} ; \omega\right), k_{-}=\operatorname{fix}\left(0.5 n_{s}\left(1-P_{c}\right)\right),
\end{aligned}
$$

in which fix $(x)$ is the integer part of real $x$.

\section{Analysis of a complex aerospace engineering system}

\subsection{Mean finite element model of the free satellite}

The mean (nominal) model of the satellite is a refined finite element model with about 120,000 DOFs, provided by the European Space Agency (ESA). It is shown in Fig. 1, where the model is rotated by $90^{\circ}$, as the $z$-axis is the vertical axis. The external applied load consists of a harmonic base excitation in the range $[5,100] \mathrm{Hz}$, prescribed in terms of the acceleration at the interface of the satellite. In Fig. 1 the interface is located on the hidden face of the structure, with the surface vector pointing in the negative $z$-axis. The prescribed acceleration is enforced with the socalled large mass approach, in which a single, large, fictitious mass is applied at the base of the structure and a proportionately large force is applied to this mass. In the present case, the node with the large mass is located at the center of the circular interface ring and is connected with the interface nodes through rigid beam elements. To avoid unwanted interface rotations, large mass moments of inertia in the order of the mass have been assigned to the point mass, too. In the range $[5,25] \mathrm{Hz}$, the imposed acceleration is $1 \mathrm{~g}$ and in the range $[25,100] \mathrm{Hz}$, the acceleration amplitude is reduced to $0.8 \mathrm{~g}$. A frequency response analysis has been carried out for the range $[5,100] \mathrm{Hz}$. The mean damping ratios $\underline{\xi}_{j}=0.015$ and $\underline{\xi}_{j}=0.025$, for $j=1, \ldots, M$ have been assumed for frequencies below $30 \mathrm{~Hz}$ and for frequencies above $30 \mathrm{~Hz}$ respectively.

\subsection{Data for the parametric probabilistic approach}

In the parametric probabilistic model, the uncertain parameters of the mean finite element model are modeled by random variables. This relates both to material and geometric properties of the 
satellite finite element model, such as beam section dimensions, composite material fiber orientation, non structural masses, elastic moduli etc. For such a complex structure, it is very difficult to make an a priori assessment of whether the uncertainty in a given parameter is influential and should be considered or if it can be neglected. Therefore, the parameters have been classified into various types and for each occurence of a certain parameter type, an independent random variable has been defined. The assumed probability distribution and the magnitude of the variability depend on the parameter type and are reported in Table 1 in Appendix A. For instance, for Young's moduli of isotropic materials, the coefficient of variation $\sigma / \mu$ was assumed to be 0.08. In summary this approach leads to a total of 1319 independent random variables with coefficient of variations between 0.04 and 0.12 . Moreover, in order to adequately account for the significant uncertainty associated with damping, the coefficient of variation for damping has been assumed to be 0.4 with a log-normal distribution. It should be noted that the magnitude of the scatter has been selected on the basis of data available in the literature [23-26].

\subsection{Estimation of the dispersion parameters of the non-parametric probabilistic approach}

The probability distribution $p_{\Omega_{1}^{\text {par }}}$ introduced in Section 4 is estimated with $n_{s}=1500$ realisations. Then the minimisation of function $J\left(\delta_{M}, \delta_{K}\right)$ yields $\delta_{M}=0.14217$ and $\delta_{K}=0.13487$. The Fig. 2 shows the probability distributions $\Omega \mapsto p_{\Omega_{1}^{\text {par }}}(\Omega)$ (thin line) and $\Omega \mapsto p_{\Omega_{1}^{\text {par }}}(\Omega)$ (thick line). It can be seen that the two probability density functions issued from both probabilistic approaches match reasonably well. Concerning the dispersion parameter $\delta_{D}$ whose expression is given by Eq. (18), the Fig. 3 shows the estimation of $\delta_{D}$ with respect to the number $n_{s}$ of realisations used for the Monte Carlo simulation. It can be seen that a good convergence is reached for $n_{s}=300$ and yields $\delta_{D}=0.4166$.

\subsection{Convergence analysis of the stochastic system}

The Monte Carlo numerical simulation is carried out with $n_{s}$ realisations denoted by $\theta_{1}, \ldots, \theta_{n_{s}}$. The stochastic mechanical system is studied in the low-frequency band $\mathbb{B}=[5 ; 100] \mathrm{Hz}$ with the following values of the dispersion parameters $\delta_{M}=0.1422, \delta_{D}=0.4166, \delta_{K}=0.1349$. 
The Fig. 4 displays the graph of the function $n_{s} \mapsto 20 \log _{10}\left(\operatorname{Conv}\left(n_{s}, n\right)\right)$ for $n=294$. It can be seen that a good convergence with respect to the Monte Carlo numerical simulation is obtained for $n_{s}=750$. The Fig. 5 shows the graph of the function $n \mapsto 20 \log _{10}\left(\operatorname{Conv}\left(n_{s}, n\right)\right)$ for $n_{s}=750$. It can be seen that a good approximation is obtained for $n=150$.

\subsection{Context of the analysis}

In previous works, it has been shown that the confidence region constructed with the nonparametric probabilistic approach from a mean model of a structure was able to capture the experimental frequency response function in presence of model uncertainties which was not the case for the confidence region constructed with the parametric probabilistic approach adopted to take into account data uncertainties (see for instance for theoretical explanations $[14,13]$ and for numerical and experimental validations $[10,12,15])$. These previous works observations show that the non-parametric probabilistic approach is representative of actual model uncertainties. In addition, the differences between the confidence region obtained with each probabilistic approach are justified by the fact that the parametric probabilistic approach takes into account data uncertainties while the non-parametric one takes into account both model and data uncertainties. For the complex structure investigated here, no experimental data concerning the dynamic response of the manufactured satellite is available. For such a complex aerospace system, all the internal equipments of the satellite cannot be represented by the mean finite element, inducing model uncertainties. The parametric probabilistic model is constructed in order to analyse data uncertainties.

\subsection{Confidence region estimation of the random response of the free satellite}

In order to simplify the notations, indicial exponents ${ }^{\text {npar }}$ and ${ }^{\text {par }}$ are omitted.

One considers the random response of the free satellite at the node $j_{\text {obs }}$ (see Fig. 1) in low frequency band $\mathbb{B}$. The numerical calculations are carried out with $n=150$ and $n_{s}=1500$. Figs. 6 and 7 display the graphs of the confidence region of the random displacements of the 
node $j_{\text {obs }}$ obtained for a probability level equal to 0.96 and constructed with the quantile method. The thick dashed-dotted line shows the graph $\nu \mapsto \underline{w}_{j \text { obs }}(\nu)$, in which $\nu=\omega /(2 \pi)$ is the circular frequency in Hertz. The thin dotted line corresponds to $\nu \mapsto W_{j_{\mathrm{obs}}}^{0}(\nu)$. The confidence region corresponds to the gray filled zone whose envelopes are delimited by the mappings $\nu \mapsto$ $w_{j_{\mathrm{obs}}}^{-}(\nu)$ and $\nu \mapsto w_{j_{\mathrm{obs}}}^{+}(\nu)$ and calculated for the frequency band $[15,100] \mathrm{Hz}$. Fig. 6 corresponds to the parametric probabilistic modelling whereas Fig. 7 corresponds to the non-parametric probabilistic modelling. From Figs. 6 and 7, it can be seen that the confidence region obtained are similar for frequencies lower than $25 \mathrm{~Hz}$ which means that model uncertainties are small with respect to data uncertainties in this low frequency band. For frequencies greater than $25 \mathrm{~Hz}$, Figs. 6 and 7 show that model uncertainties are significant and increase with the frequency. In addition, it should be noted that the mean of the random response obtained with the nonparametric approach is very different from the response of the mean model. Furthermore, there exist frequencies for which the response of the mean model is outside from the confidence region. On the contrary, this phenomenon is not present for the random response obtained with the parametric probabilistic model displayed in Fig. 6. This is due to the fact that the nonparametric probabilistic approach has the capability to take into account model uncertainties and not only data uncertainties. From these results, it can be concluded that the numerical model of the free satellite is robust to data and model uncertainties in frequency band $[15,25] \mathrm{Hz}$, stays robust to data uncertainties in $[25,58] \mathrm{Hz}$ and is not robust to model uncertainties in the frequency band $[25,100] \mathrm{Hz}$.

\subsection{Mean finite element model of the satellite coupled with its launcher}

The mean model of the coupled mechanical system is three dimensional finite element model. The finite element model of the launcher is decomposed in four subdomains which are two symmetric solid propellant boosters (EAPM, EAPP), a main stage with liquid propellant tanks (EPC) and the upper composite (UC) which contains the satellite. The assembled finite element model of the mechanical system is shown in Fig. 8. The coupled launcher-satellite system is subjected to a deterministic excitation which corresponds to a pressure oscillation distributed along the longitudinal direction of the each booster with opposite phases. 
6.8 Confidence region estimation of the random response of the coupled launcher-satellite system

In the present case, the launcher is assumed to be without uncertainties while the satellite has uncertainties. Hence, the uncertainties in the coupled system are nonhomogeneous. The implementation of the non-parametric probabilistic approach for nonhomogeneous uncertainties requires to describe the mechanical system by dynamic substructuring [10]. The coupled system is constituted of five substructures. The dynamic substructuring method used is the Craig and Bampton modal synthesis method [27].

For the non-parametric probabilistic approach of uncertainties in the solution for the coupled system, the numerical analysis is carried out in the low frequency band $[5,54] \mathrm{Hz}$ with $n_{s}=1500$. The dispersion parameters for the solution are those defined in Section 6.4. Fig. 9 displays the graph of the confidence region for the random displacements at the node $j_{\text {obs }}$ with a probability level equal to 0.96 . The thick dashed-dotted line shows the graph $\nu \mapsto \underline{w}_{j_{\text {obs }}}(\nu)$ with $\nu=\omega /(2 \pi)$ the circular frequency. The thin dotted line corresponds to $\nu \mapsto W_{j_{\text {obs }}}^{0}(\nu)$. The confidence region corresponds to the gray filled zone whose envelop are delimited by the mappings $\nu \mapsto w_{j_{\mathrm{obs}}}^{-}(\nu)$ and $\nu \mapsto w_{j_{\mathrm{obs}}}^{+}(\nu)$. Fig. 9 shows that the larger the frequency, the larger the confidence region, which is coherent with the fact that the sensitivity of model uncertainties increases with frequency. Again two frequency zones can be distinguished. For frequencies lower than $30 \mathrm{~Hz}$, the confidence region is narrow and centered around the response of the mean model. This means that the numerical model of the coupled system is robust to model and data uncertainties in the satellite. On the other hand, Fig. 9 shows that the sensitivity to model and data uncertainties increase with the frequency and is significant in frequency band $[30,54] \mathrm{Hz}$. Then, a gap between the mean of the random response and between the response of the mean model appears at $30 \mathrm{~Hz}$, and goes on increasing with respect to the frequency. Consequently, the random response seems to be very sensitive to model uncertainties for frequencies greater than $30 \mathrm{~Hz}$. 
6.9 Robustness of the numerical model of the coupled system with respect to model and data uncertainties

The analysis is performed in the frequency band $[30,54] \mathrm{Hz}$ for the parametric and the nonparametric probabilistic approaches. Fig. 10 displays the confidence region constructed with the parametric probabilistic approach and Fig. 11 with the non-parametric probabilistic approach. Fig. 10 shows that the random response of the satellite coupled with the launcher is more robust with respect to data uncertainties in the frequency band $[44,54] \mathrm{Hz}$ than in the frequency band $[36,44] \mathrm{Hz}$. Fig. 11 shows that the robustness of the response is small with respect to model uncertainties in the frequency band $[36,54] \mathrm{Hz}$. It should be noted that the response is more sensitive to model uncertainties in the frequency band $[44,54] \mathrm{Hz}$ than in the frequency band $[36,44] \mathrm{Hz}$.

\section{Synthesis of the results and conclusion}

Although the dispersion level related to the random uncertainties of the satellite is the same for both probabilistic approaches, the random forced responses do not look similar. The aim of this paper is not to compare these responses because the two probabilistic approaches do not model the same kind of random uncertainties. The parametric one models data uncertainties while the non-parametric one models both data and model uncertainties. In the frequency band $[15,25 \mathrm{~Hz}]$ (for the free satellite) or $[5,30 \mathrm{~Hz}]$ (for the coupled launcher-satellite system), the numerical model is relatively robust with respect to data uncertainties and to model uncertainties and, in addition is not really sensitive to model uncertainties, data uncertainties being preponderant. For higher frequencies, [25, 100 Hz] (for the free satellite) or [30, $54 \mathrm{~Hz}]$ (for the coupled launcher-satellite system), it can be seen that the numerical model is not robust to model uncertainties. The results show then that the numerical model used for the satellite and the coupled launcher-satellite system are more sensitive to model uncertainties than to data uncertainties. Hence, it can concluded that the two probabilistic approaches are complementary. The parametric probabilistic approach is useful to estimate the robustness with respect to the 
data uncertainties. Both parametric and non-parametric probabilistic approaches are required to give an estimation of the robustness with respect to model uncertainties.

\section{Acknowledgements}

The authors thank the European Space Research and Technology Center (ESTEC) of the European Space Agency (ESA) for providing the satellite and the Ariane 5 launcher finite element models. The partial financial support by the AMADEUS european program (Projects $\mathrm{N}^{\circ}$ 22/2003 and LAM03) is gratefully acknowledged. 


\section{Appendix A Assumptions for the parametric probabilistic approach}

Table 1 shows the assumptions made for the random variables representing the uncertain model parameters in the parametric probabilistic approach. Specifically, the table specifies the distribution type and the magnitude of the scatter, expressed in terms of the coefficient of variation (C.o.V.), i.e. the ratio between standard deviation $\sigma$ and the mean $\mu$. An exception is made for the fiber orientation angle, where the mean is zero and the C.o.V. thus not defined; the standard deviation is provided instead. A truncated normal distribution has been assumed for all uncertain parameters, with the exception of the damping parameters modeled by a log-normal distribution. 


\section{References}

[1] R. Ibrahim, Structural dynamics with parameter uncertainties, Applied Mechanical Reviews 40 (3) (1987) 309-328.

[2] C. Bucher, C. Brenner, Stochastic response of uncertain systems, Archive of Applied Mechanics 62 (8) (1992) 507-516.

[3] R. Singh, C. Lee, Frequency response of linear systems with parameter uncertainties, Journal of Sound and Vibration 168 (1) (1993) 507-516.

[4] Y. Lin, G. Cai, Probabilistic structural dynamics, Mc-Graw Hill, 1995.

[5] G. Schuëller (Editor), A state-of-the-art report on computational stochastic mechanics, Probabilistic Engineering Mechanics 12 (4) (1997) 197-321.

[6] G. Schuëller, Computational stochastic mechanics, recent advances, Computers \& Structures 79 (2001) 2225-2234.

[7] C. Schenk, G. Schuëller, Buckling analysis of cylindrical shells with random geometric imperfections, International Journal of Nonlinear Mechanics 38 (7) (2003) 1119-1132.

[8] C. Soize, A nonparametric model of random uncertainties for reduced matrix models in structural dynamics, Probabilistic Engineering Mechanics 15 (3) (2000) 277-294.

[9] C. Soize, Maximum entropy approach for modeling random uncertainties in transient elastodynamics., Journal of the Acoustical Society of America 109 (5) (2001) 1979-1996.

[10] H. Chebli, C. Soize, Experimental validation of a nonparametric probabilistic model of nonhomogeneous uncertainties for dynamical systems, Journal of the Acoustical Society of America 115 (2) (2004) 697-705.

[11] J. Duchereau, C. Soize, Transient dynamics in structures with nonhomogeneous uncertainties induced by complex joints (in press), Mechanical Systems and Signal Processing.

[12] C. Chen, D. Duhamel, C. Soize, Uncertainties in structural dynamics for composite sandwich panels, in: the Proceedings (CD-ROM) of OSMA 2004, the International Conference on Noise and Vibration Engineering, Katholieke Universiteit Leuven, Belgium, September 20-22, 2004. CDROM edited by the Katholieke Universiteit Leuven, ISBN 90-73-802-82-2. 
[13] C. Soize, Random matrix theory for modeling random uncertainties in computational mechanics (in press), Computer Methods in Applied Mechanics and Engineering 12 (16) (2005) 1333-1366.

[14] C. Soize, A comprehensive overview of a non-parametric probabilistic approach of model uncertainties for predictive models in structural dynamics, Journal of Sound and Vibration 288 (3) (2005) 623-652.

[15] C. Soize, Probabilistic models for computational stochastic mechanics and applications, in: Proceedings on the 9th International Conference on Structural Safety and Reliability ICOSSAR'05, Rome, Italy, 19-23 June 2005, G. Augusti, G.I. Schueller and M. Ciampoli, Millpress, Rotterdam, Netherlands, ISBN 905966040 4, 2005.

[16] E. Capiez-Lernout, C. Soize, Nonparametric modeling of random uncertainties for dynamic response of mistuned bladed-disks., ASME Journal of Engineering for Gas Turbines and Power 126 (3) (2004) 610-618.

[17] E. Capiez-Lernout, C. Soize, J.-P. Lombard, C. Dupont, E. Seinturier, Blade manufacturing tolerances definition for a mistuned industrial bladed disk, ASME Journal of Engineering for Gas Turbines and Power 127 (3) (2005) 621-628.

[18] M. Shinozuka, C. Astill, Random eigenvalue problems in structural analysis, AIAA Journal 10 (4) (1972) 456-462.

[19] H. J. Pradlwarter, G. I. Schuëller, Stochastic response evaluation of large FE-models with nonlinear hysteretic elements by complex mode synthesis, in: P. D. Spanos (Ed.), Proceedings Third International Conference on Computational Stochastic Mechanics, Balkema, Rotterdam, TheraSantorini, Greece, 1999, pp. 551-558.

[20] G. Székely, G. Schuëller, Computational procedure for a fast calculation of eigenvectors and eigenvalues of structures with random properties, Computer Methods in Applied Mechanics and Engineering 191 (8-10) (2001) 799-816.

[21] H. Pradlwarter, G. Schuëller, G. Székely, Random eigenvalue problems for large systems, Computers \& Structures 80 (27-30) (2002) 2415-2424.

[22] C. Shannon, A mathematical theory of communication, Bell System Technology Journal (1948) $379-423$ and 623-659. 
[23] P. Esnault, M. Klein, Factors of safety and reliability - present guidelines \& future aspects, in: Proc. Conference on Spacecraft Structures, Materials \& Mechanical Testing, Grand Hotel Huis ter Duin, Nordwijk, The Netherlands, 1996, eSA SP-386, June 1996.

[24] M. Klein, G. Schuëller, P. Deymarie, M. Macke, P. Courrian, R. S. Capitanio, Probabilistic approach to structural factors of safety in aerospace, in: Proceedings of the International Conference on Spacecraft Structures and Mechanical Testing, Cépadués-Editions, Paris, France, 1994, pp. 679 693.

[25] G. Székely, W. Teichert, C. Brenner, H. Pradlwarter, M. Klein, G. Schuëller, Practical procedures for reliability estimation of spacecraft structures and their components, AIAA Journal 36 (8) (1998) $1509-1515$.

[26] S. Simonian, Survey of spacecraft damping measurements: Applications to electro-optic jitter problems, in: The Role of Damping in Vibration and Noise Control, Vol. DE-Vol. 5 of ASME Publication, 1987, pp. 287-292.

[27] R. Craig, M. Bampton, Coupling of substructures for dynamic analyses, AIAA Journal 6 (7) (1968) 1313-1319. 
Table 1

Table of random properties of uncertain parameters for modeling data uncertainties in the parametric probabilistic approach. 
Figure 1

Finite element model of the satellite.

Figure 2

Identification of dispersion parameters $\delta_{M}$ and $\delta_{K}$ : Graph of the probability distributions $\Omega \mapsto$ $p_{\Omega_{1}^{\mathrm{par}}}(\Omega)$ for the parametric approach (thin line) and $\Omega \mapsto p_{\Omega_{1}^{\text {narar }}}(\Omega)$ for the non-parametric approach (thick line) with dispersion parameters such that $\delta_{M}=0.14217$ and $\delta_{K}=0.13487$. Horizontal axis $\Omega$ in rad.s $\mathrm{s}^{-1}$.

Figure 3

Identification of dispersion parameter $\delta_{D}$ : Graph of $n_{s} \mapsto \delta_{D}$.

Figure 4

Convergence analysis : Graph of the function $n \mapsto 20 \log _{10}\left\{\operatorname{Conv}\left(n_{s}, n\right)\right\}$ for the stochastic system with $n_{s}=750$.

Figure 5

Convergence analysis : Graph of the function $n \mapsto 20 \log _{10}\left\{\operatorname{Conv}\left(n_{s}, n\right)\right\}$ for the stochastic system with $n_{s}=750$.

Figure 6

Confidence region of random displacement related to the node $j_{\text {obs }}$ (in $\mathrm{dB}$ ) over a low frequency band $\mathbb{B}=[15,100] \mathrm{Hz}$ and obtained with the parametric probabilistic approach: deterministic response of the mean model (thick dashed-dotted line), mean of the random response for the stochastic model (mid thin dotted line), lower and upper envelopes of the confidence region corresponding to a probability level equal to 0.96 (dark gray filled zone).

Figure 7

Confidence region of random displacement related to the node $j_{\text {obs }}$ (in $\mathrm{dB}$ ) over a low frequency 
band $\mathbb{B}=[15,100] \mathrm{Hz}$ and obtained with the non-parametric probabilistic approach: deterministic response of the mean model (thick dashed-dotted line), mean of the random response for the stochastic model (mid thin dotted line), lower and upper envelopes of the confidence region corresponding to a probability level equal to 0.96 (dark gray filled zone).

Figure 8

Finite element model of the coupled launcher-satellite structure.

Figure 9

Confidence region of random displacement related to the node $j_{\mathrm{obs}}$ (in $\mathrm{dB}$ ) over the low frequency band $[5,54] \mathrm{Hz}$ and obtained with the non-parametric probabilistic approach: deterministic response of the mean model (thick dashed-dotted line), mean of the random response for the stochastic model (thin dotted line), lower and upper envelopes of the confidence region corresponding to a probability level equal to 0.96 (dark gray filled zone).

Figure 10

Confidence region of random displacement related to the node $j_{\text {obs }}$ (in $\mathrm{dB}$ ) over the low frequency band $[30,54] \mathrm{Hz}$ and obtained with the parametric probabilistic approach: deterministic response of the mean model (thick dashed-dotted line), mean of the random response for the stochastic model (thin dotted line), lower and upper envelopes of the confidence region corresponding to a probability level equal to 0.96 (dark gray filled zone).

Figure 11

Confidence region of random displacement related to the node $j_{\text {obs }}$ (in $\mathrm{dB}$ ) over the low frequency band $[30,54] \mathrm{Hz}$ and obtained with the non-parametric probabilistic approach: deterministic response of the mean model (thick dashed-dotted line), mean of the random response for the stochastic model (thin dotted line), lower and upper envelopes of the confidence region corresponding to a probability level equal to 0.96 (dark gray filled zone). 


\begin{tabular}{|l|l|l|l|}
\hline Element/Material Type & Property & C.o.V. $(\sigma / \mu)$ & Probability Distribution \\
\hline Isotropic Material & Young's modulus & $8 \%$ & truncated Gaussian \\
& Poisson's ratio & $3 \%$ & \\
& Shear modulus & $12 \%$ & \\
& Mass density & $4 \%$ & \\
\hline Orthotropic Shell & Young's modulus & $8 \%$ & truncated Gaussian \\
& Poisson's ratio & $3 \%$ & \\
& Shear modulus & $12 \%$ & \\
& Mass density & $4 \%$ & \\
\hline Solid Element Anisotropic & Mat. property matrix & $12 \%$ & truncated Gaussian \\
Isotropic Material & Mass density & $4 \%$ & \\
\hline Simple Beam & Section dimension & $5 \%$ & truncated Gaussian \\
& Non-structural mass & $8 \%$ & \\
\hline Layered Composite & Non-structural Mass & $8 \%$ & truncated Gaussian \\
Material & Thickness of plies & $12 \%$ & \\
\hline Spring element property & Orientation angle & $\sigma=1.5$ & \\
\hline Shell element & Elastic prop. value & $8 \%$ & truncated Gaussian \\
\hline Spring element & Membrane Thickness & $4 \%$ & truncated Gaussian \\
\hline Concentrated mass & Non-structural Mass & $8 \%$ & \\
\hline Damping & Stiffness & $10 \%$ & truncated Gaussian \\
\hline & Mass & $3 \%$ & truncated Gaussian \\
\hline & Modal Damping & $40 \%$ & lognormal \\
\hline & Structural Damping & $25 \%$ & \\
\hline
\end{tabular}

Table 1

Table of random properties of uncertain parameters for modeling data uncertainties in the parametric probabilistic approach. 

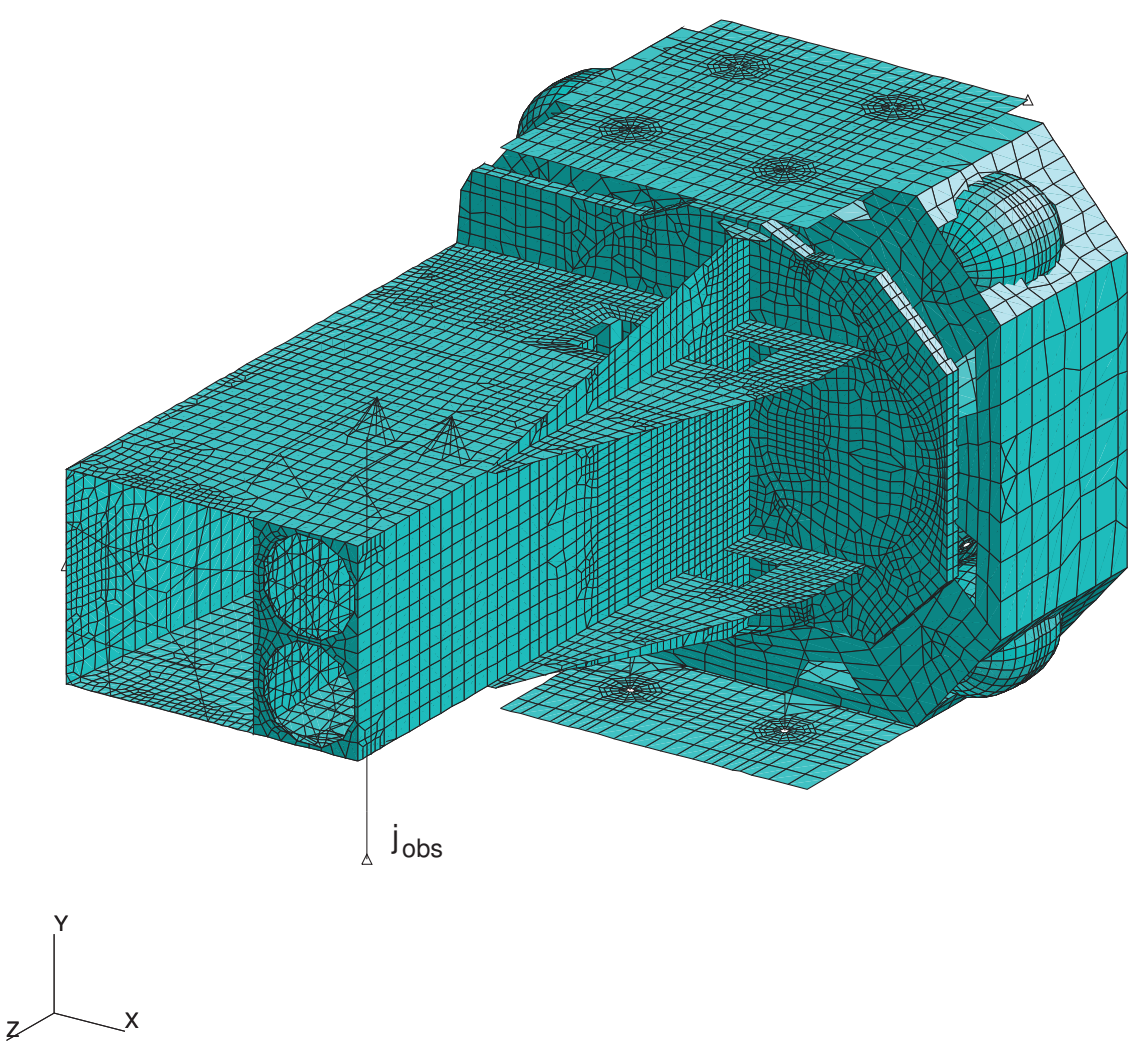

Fig. 1. Finite element model of the satellite. 


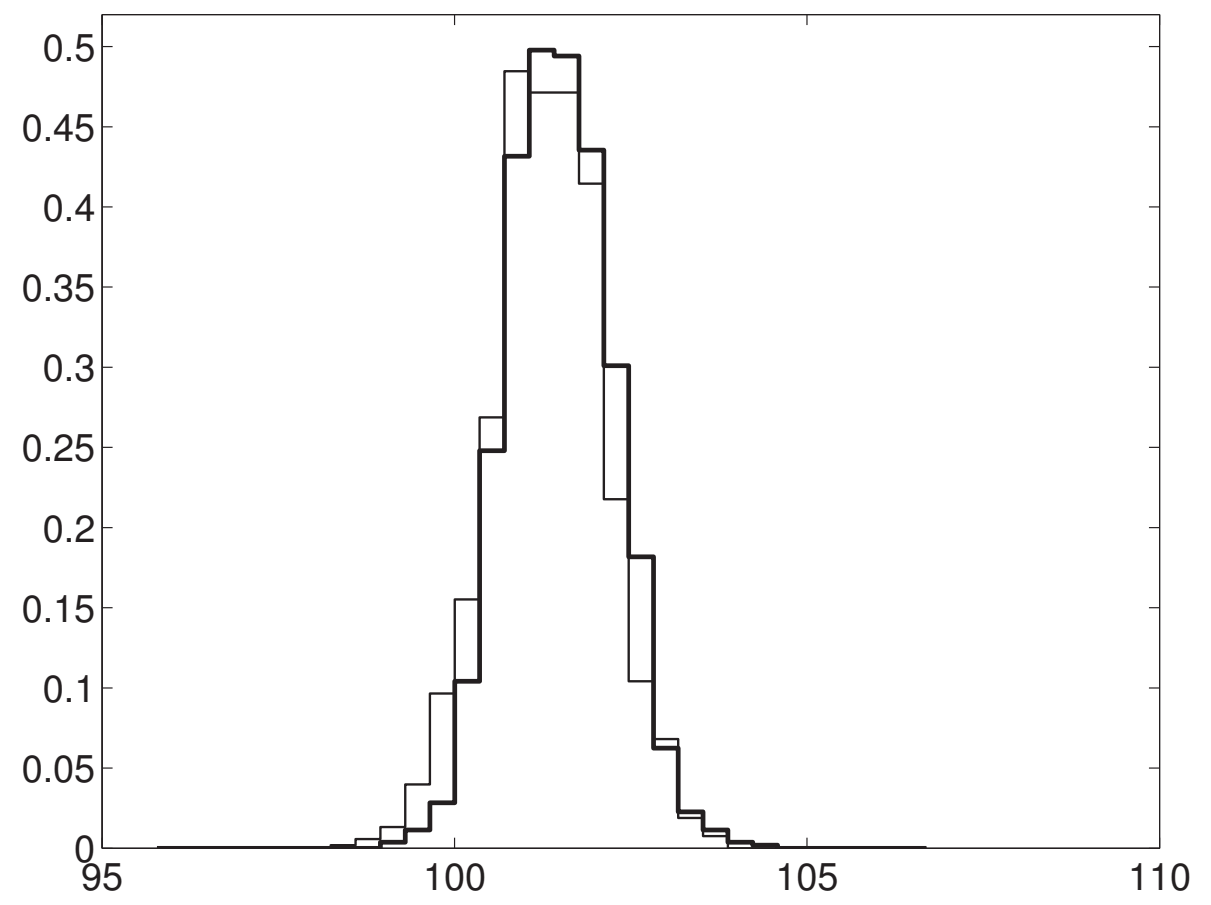

Fig. 2. Identification of dispersion parameters $\delta_{M}$ and $\delta_{K}$ : Graph of the probability distributions $\Omega \mapsto p_{\Omega_{1}^{\mathrm{par}}}(\Omega)$ for the parametric approach (thin line) and $\Omega \mapsto p_{\Omega_{1}^{\text {npar }}}(\Omega)$ for the non-parametric approach (thick line) with dispersion parameters such that $\delta_{M}=0.14217$ and $\delta_{K}=0.13487$. Horizontal axis $\Omega$ in rad.s ${ }^{-1}$. 


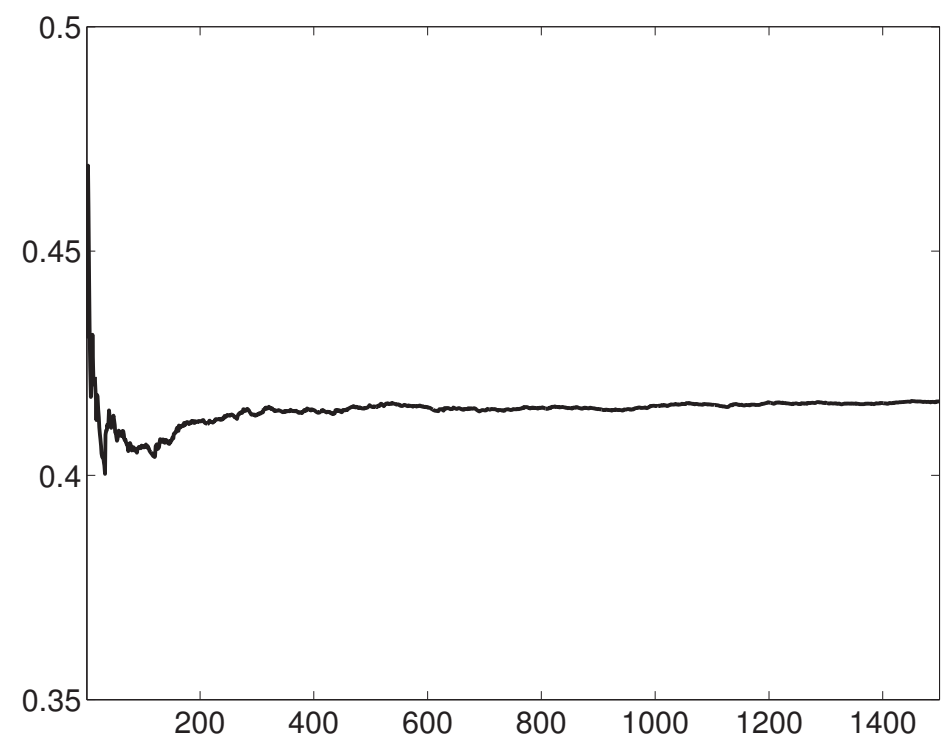

Fig. 3. Identification of dispersion parameter $\delta_{D}$ : Graph of $n_{s} \mapsto \delta_{D}$. 


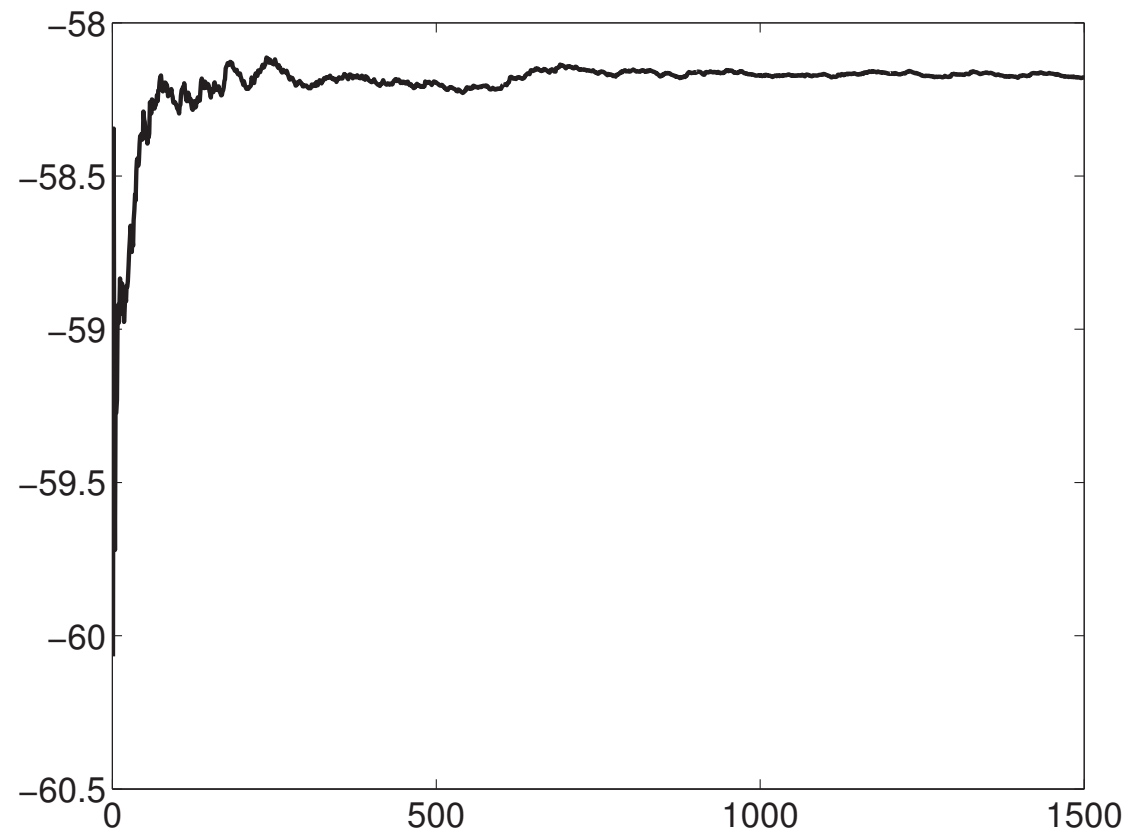

Fig. 4. Convergence analysis: Graph of the function $n_{s} \mapsto 20 \log _{10}\left\{\operatorname{Conv}\left(n_{s}, n\right)\right\}$ for the stochastic system with $n=294$. 


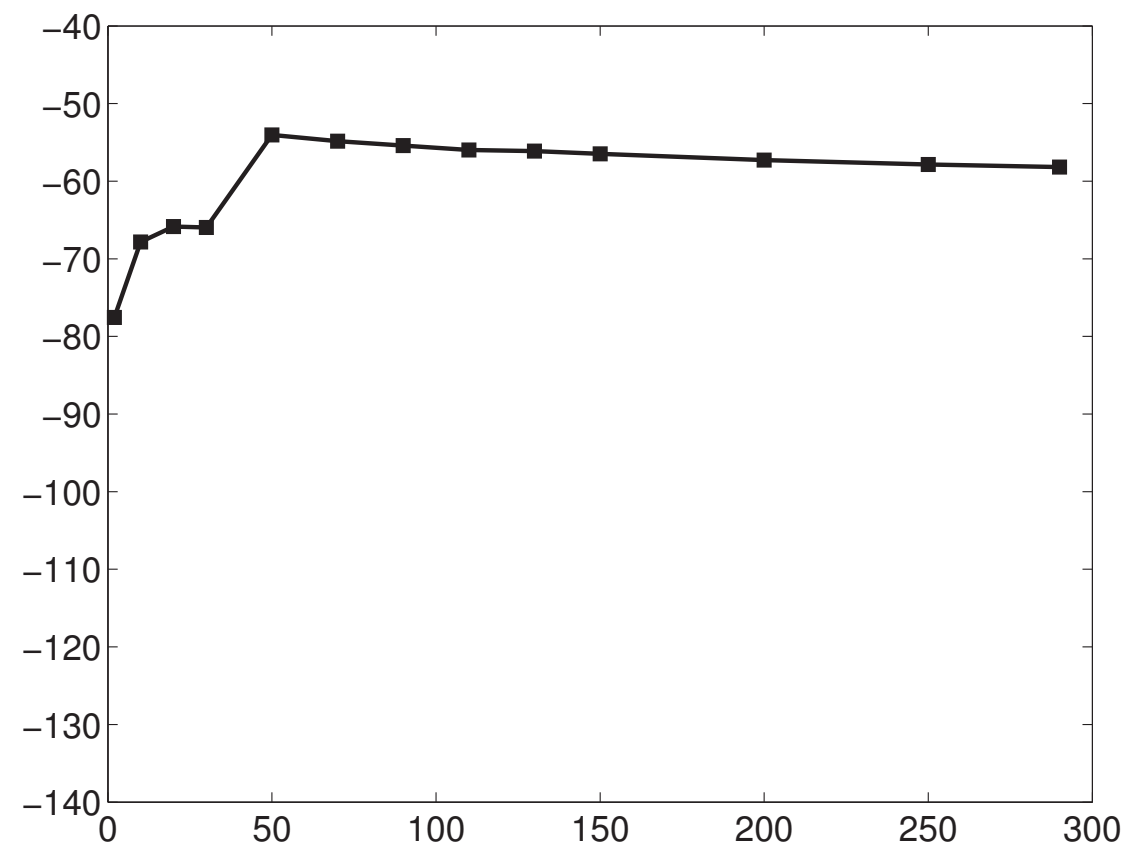

Fig. 5. Convergence analysis : Graph of the function $n \mapsto 20 \log _{10}\left\{\operatorname{Conv}\left(n_{s}, n\right)\right\}$ for the stochastic system with $n_{s}=750$. 


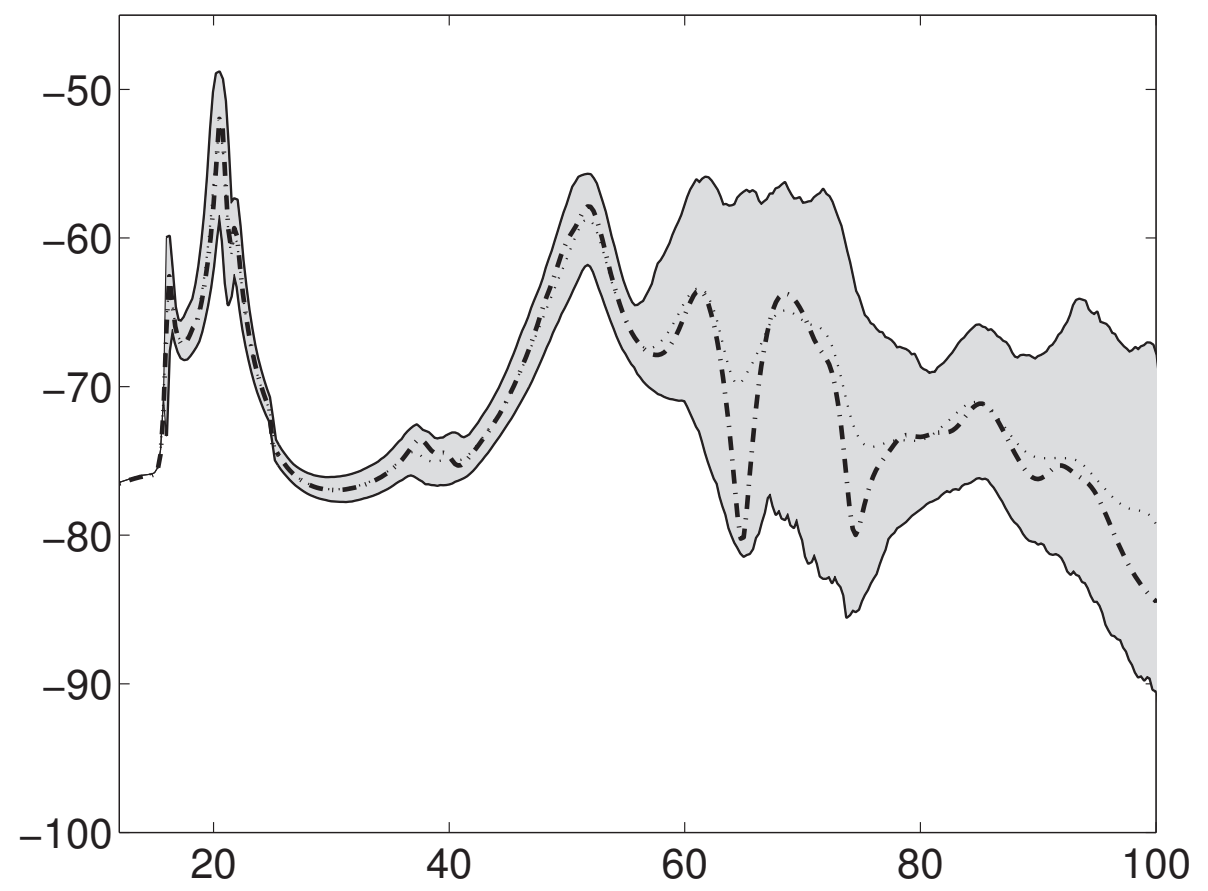

Fig. 6. Confidence region of random displacement related to the node $j_{\text {obs }}$ (in $\mathrm{dB}$ ) over a low frequency band $\mathbb{B}=[15,100] \mathrm{Hz}$ and obtained with the parametric probabilistic approach: deterministic response of the mean model (thick dashed-dotted line), mean of the random response for the stochastic model (thin dotted line), lower and upper envelopes of the confidence region corresponding to a probability level equal to 0.96 (dark gray filled zone). 


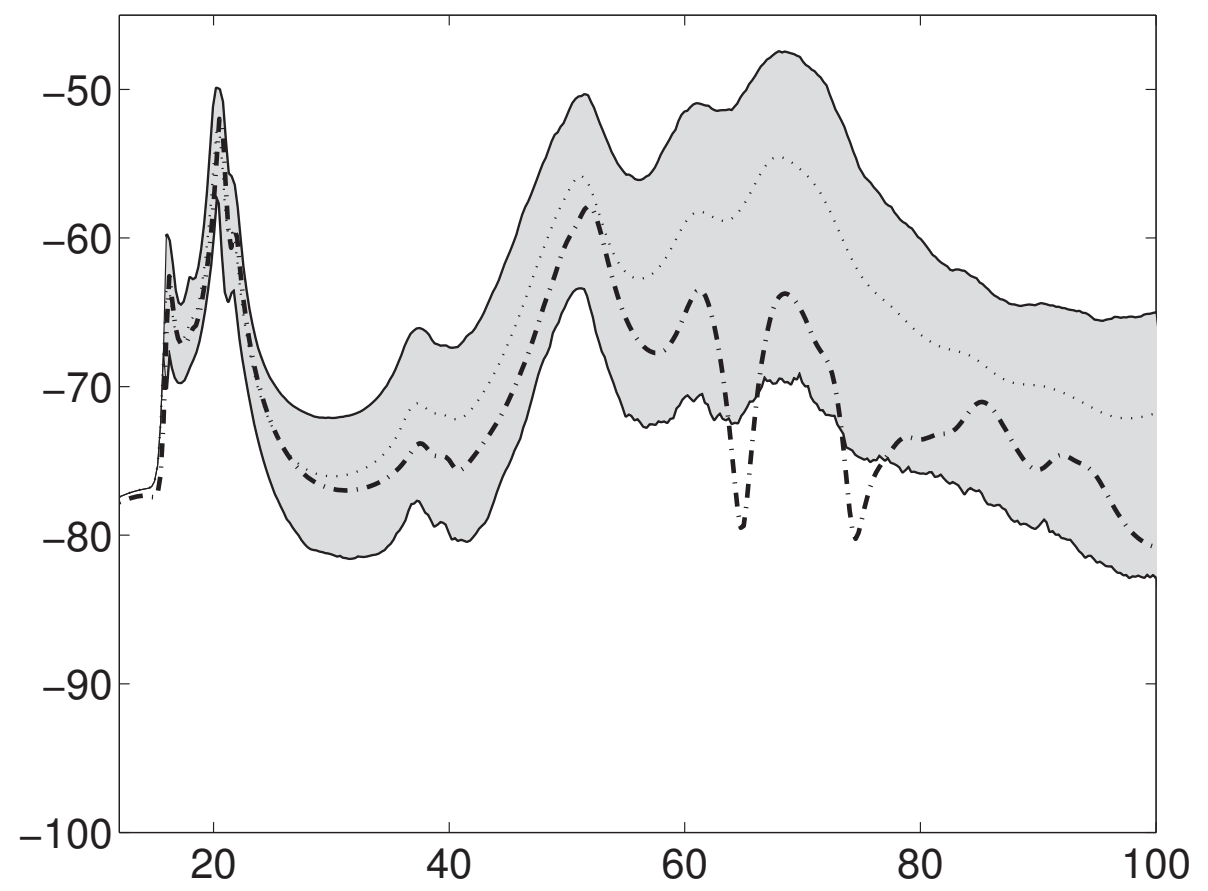

Fig. 7. Confidence region of random displacement related to the node $j_{\text {obs }}$ (in $\mathrm{dB}$ ) over a low frequency band $\mathbb{B}=[15,100] \mathrm{Hz}$ and obtained with the non-parametric probabilistic approach: deterministic response of the mean model (thick dashed-dotted line), mean of the random response for the stochastic model (mid thin dotted line), lower and upper envelopes of the confidence region corresponding to a probability level equal to 0.96 (dark gray filled zone). 


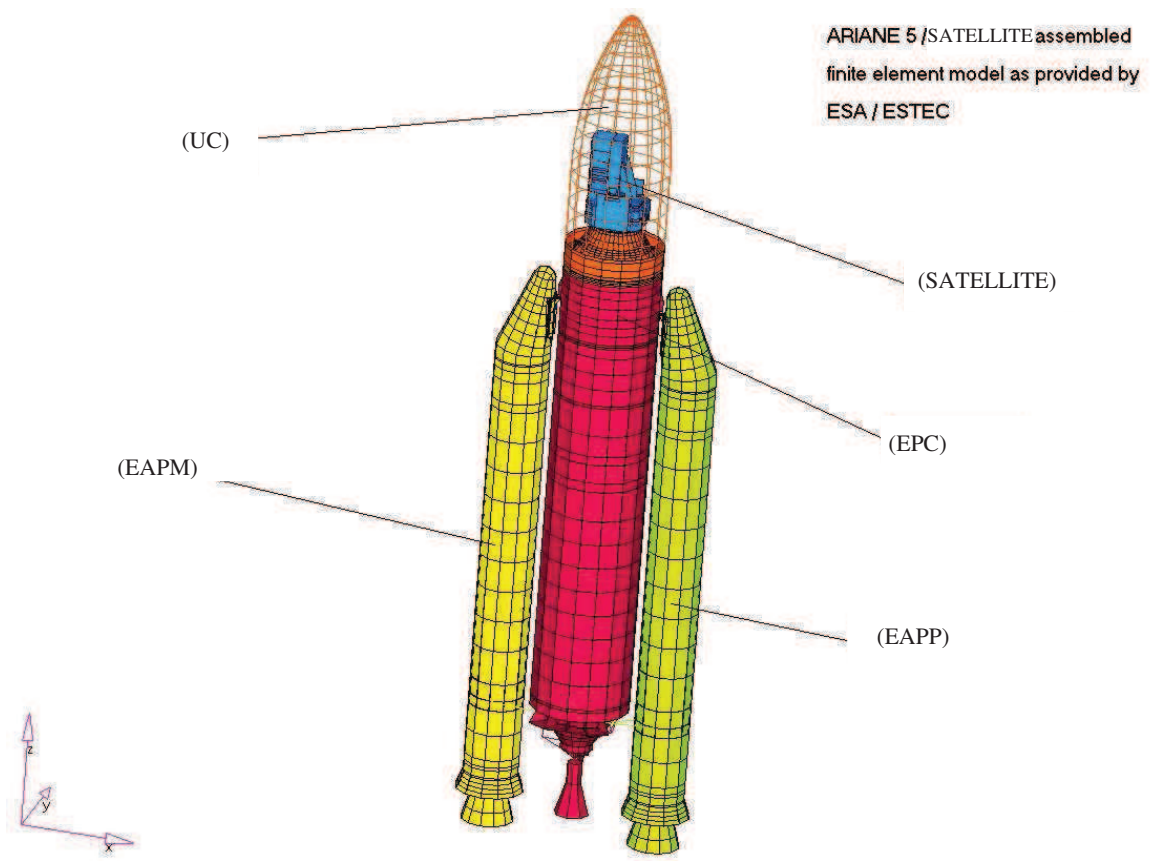

Fig. 8. Finite element model of the coupled launcher-satellite structure. 


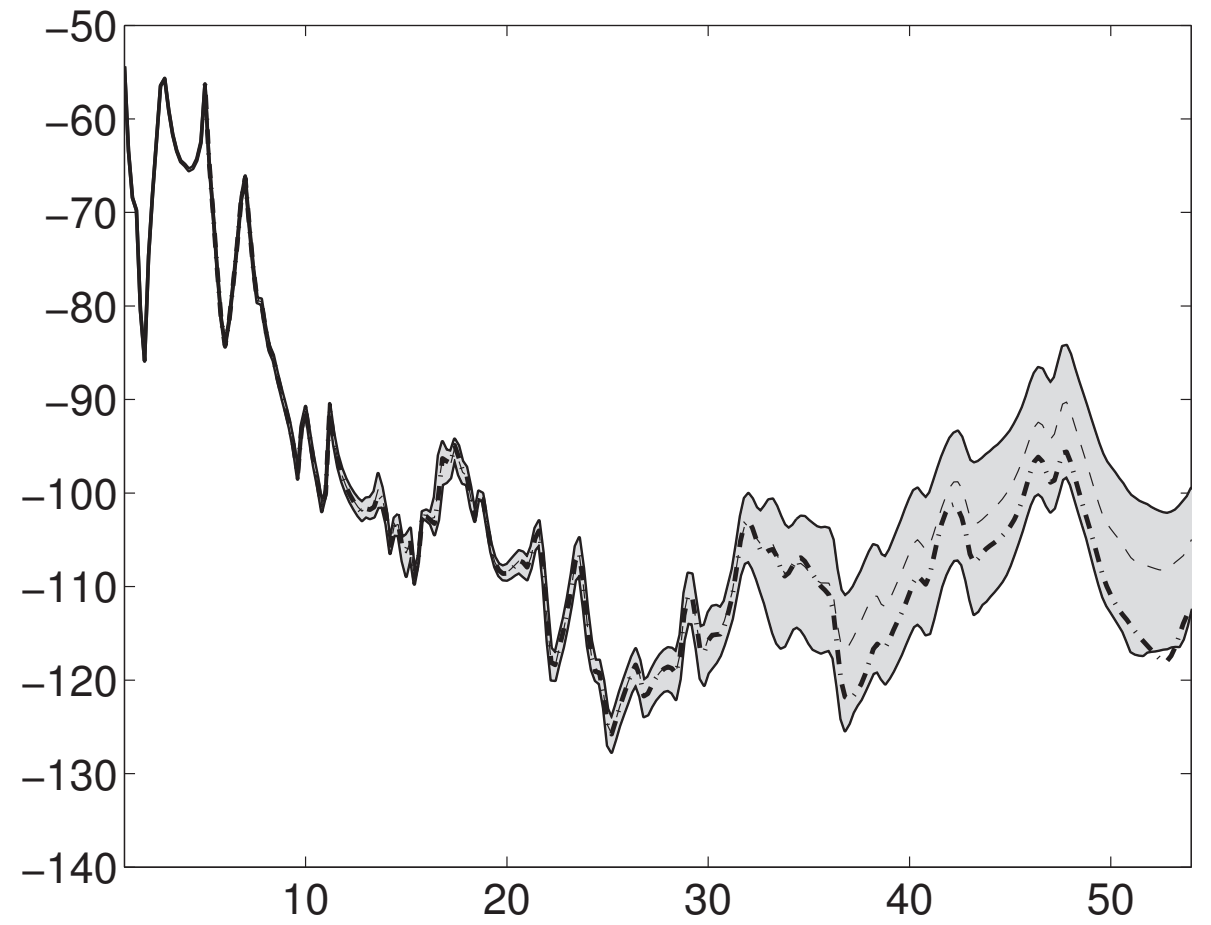

Fig. 9. Confidence region of random displacement related to the node $j_{\text {obs }}$ (in $\mathrm{dB}$ ) over the low frequency band $[5,54] \mathrm{Hz}$ and obtained with the non-parametric probabilistic approach: deterministic response of the mean model (thick dashed-dotted line), mean of the random response for the stochastic model (thin dotted line), lower and upper envelopes of the confidence region corresponding to a probability level equal to 0.96 (dark gray filled zone). 


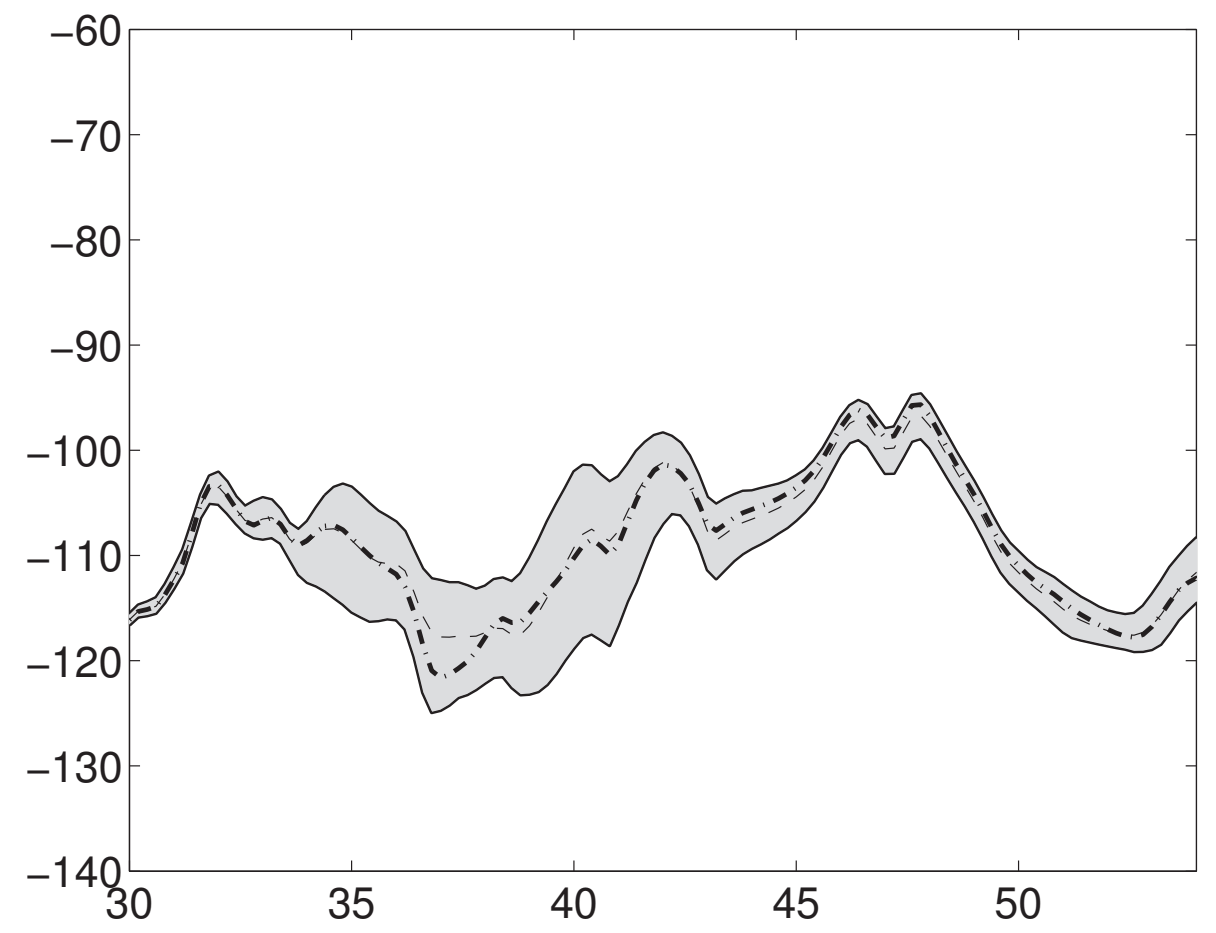

Fig. 10. Confidence region of random displacement related to the node $j_{\text {obs }}$ (in $\mathrm{dB}$ ) over the low frequency band $[30,54] \mathrm{Hz}$ and obtained with the parametric probabilistic approach: deterministic response of the mean model (thick dashed-dotted line), mean of the random response for the stochastic model (thin dotted line), lower and upper envelopes of the confidence region corresponding to a probability level equal to 0.96 (dark gray filled zone). 


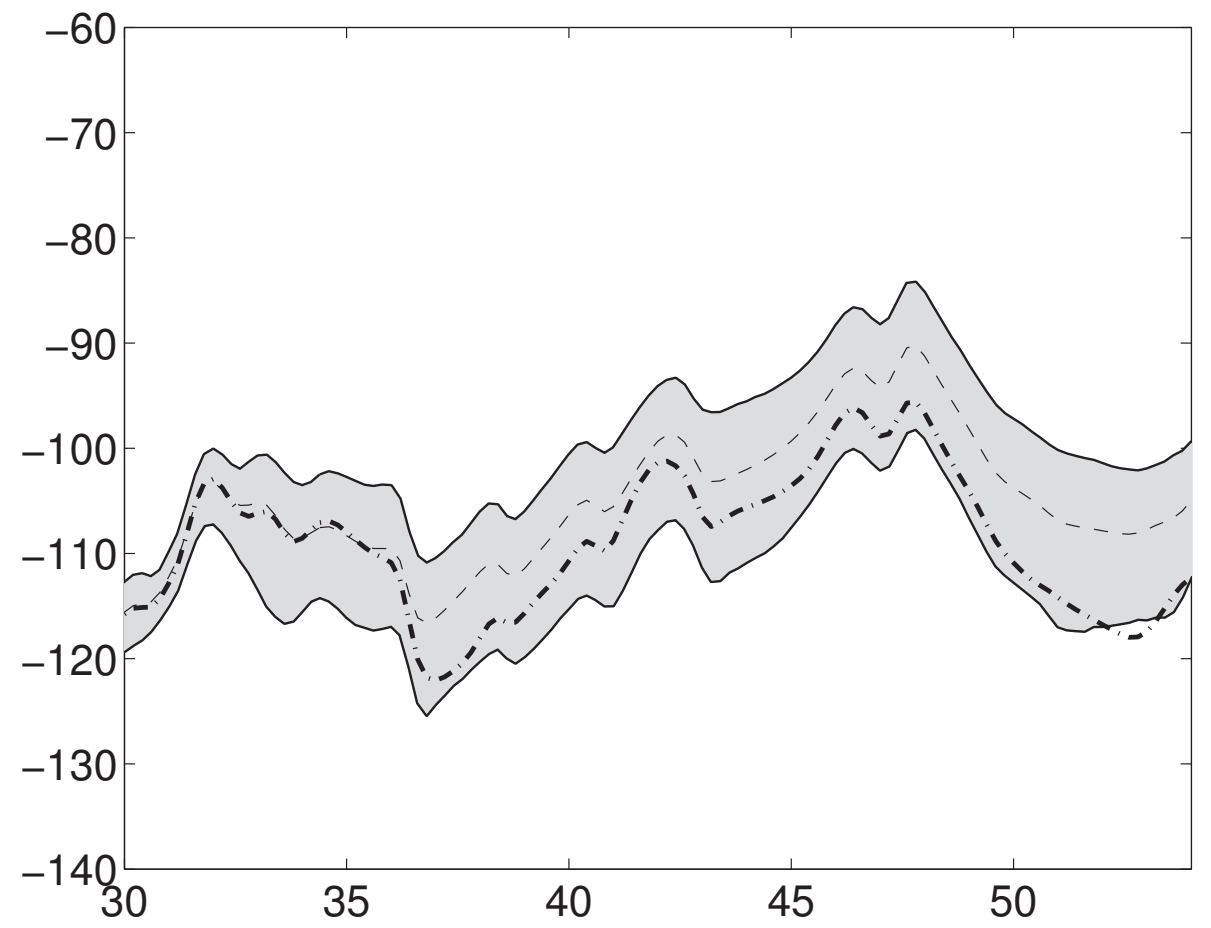

Fig. 11. Confidence region of random displacement related to the node $j_{\text {obs }}$ (in $\mathrm{dB}$ ) over the low frequency band $[30,54] \mathrm{Hz}$ and obtained with the non-parametric probabilistic approach: deterministic response of the mean model (thick dashed-dotted line), mean of the random response for the stochastic model (thin dotted line), lower and upper envelopes of the confidence region corresponding to a probability level equal to 0.96 (dark gray filled zone). 